\title{
Techniques for Maintaining Population Diversity in Classical and Agent-Based Multi-objective Evolutionary Algorithms
}

\author{
Rafał Dreżewski and Leszek Siwik \\ Department of Computer Science \\ AGH University of Science and Technology, Kraków, Poland \\ \{drezew, siwik\}@agh.edu.pl
}

\begin{abstract}
The loss of population diversity is one of the main problems in some applications of evolutionary algorithms. In order to maintain useful population diversity some special techniques must be used, like niching or co-evolutionary mechanisms. In this paper the mechanisms for maintaining population diversity in agent-based multi-objective (co-)evolutionary algorithms are proposed. The presentation of techniques is accompanied by the results of experiments and comparisons to "classical" evolutionary multi-objective algorithms.
\end{abstract}

Keywords: Agent-based evolutionary computation, maintaining population diversity, sexual selection, flock-based operators.

\section{Introduction}

Evolutionary Algorithms (EAs) are techniques inspired by Darwinian model of evolutionary processes observed in nature. They have demonstrated in practice efficiency and robustness as global optimization techniques. However, sometimes the loss of useful population diversity limits the possibilities of their application in some areas (like, for example, multi-modal optimization, multi-criteria optimization, dynamic problems, etc.)

In the case of multi-objective optimization problems loss of population diversity may cause that the population locates in the areas far from Pareto frontier or that individuals are located only in selected areas of Pareto frontier. In the case of multi-objective problems with many local Pareto frontiers (defined by Deb in [5]) the loss of population diversity may result in locating only local Pareto frontier instead of a global one. In order to avoid such negative tendencies special mechanisms are used, like niching techniques, co-evolutionary algorithms and sexual selection.

Evolutionary multi-agent systems (EMAS) are multi-agent systems, in which there are three basic mechanisms needed in order to start and maintain evolutionary processes: limited resources that agents need for all activities and for which they compete, and agents' abilities to reproduce and die. Basic EMAS model also suffers from the negative tendency to loss the population diversity, however, as we will show in the following sections, it can be equipped with additional mechanisms and operators which improve the quality of obtained results. The general model of co-evolutionary multi-agent system 
(CoEMAS) [6] introduces additionally the notions of species, sexes, and interactions between them. CoEMAS allows modeling and simulation of different co-evolutionary interactions, which can serve as the basis for constructing the techniques of maintaining population diversity and improving adaptive capabilities of such systems (for example see [7]).

In the following sections mechanisms for maintaining useful population diversity in "classical" evolutionary multi-objective algorithms are presented. Next, new techniques for (co-)evolutionary multi agent systems for multi-objective optimization are proposed. The presentation of proposed techniques is accompanied with the examples of selected experimental results and comparisons to "classical" multi-objective evolutionary algorithms.

\section{Previous Research on Maintaining Population Diversity in Evolutionary Multi-objective Algorithms}

In order to maintain useful population diversity and introduce speciation (process of forming species_-subpopulations-located in different areas of solutions' space) special techniques are used like niching mechanisms and co-evolutionary models. Niching techniques are primarily applied in multi-modal optimization problems, but they are also used in evolutionary multi-objective algorithms. During the years of research various niching techniques have been proposed [16], which allow niche formation via the modification of mechanism of selecting individuals for new generation (crowding model), the modification of the parent selection mechanism (fitness sharing technique or sexual selection mechanism), or restricted application of selection and/or recombination mechanisms (by grouping individuals into subpopulations or by introducing the environment with some topography in which the individuals are located).

Fitness sharing technique in objective space was used in Hajela and Lin genetic algorithm for multi-objective optimization based on weighting method [10], by Fonseca and Fleming in their multi-objective genetic algorithm using Pareto-based ranking procedure [8], and in the niched Pareto genetic algorithm (NPGA) (during the tournament selection in order to decide which individual wins the tournament) [11]. In nondominated sorting genetic algorithm (NSGA) the fitness sharing is performed in decision space, within each set of non-dominated individuals separately, in order to maintain high population diversity [17]. In strength Pareto evolutionary algorithm (SPEA) [19] special type of fitness sharing (based on Pareto domination relation) is used in order to maintain diversity.

In co-evolutionary algorithms the fitness of each individual depends not only on the quality of solution to the given problem but also (or solely) on other individuals' fitness. This makes such techniques applicable in the cases where the fitness function formulation is difficult (or even impossible). Co-evolutionary algorithms are also applicable in the cases when we want to maintain population diversity. Generally, each of the coevolutionary technique belongs to one of two classes: competitive or co-operative.

Laumanns, Rudolph and Schwefel proposed co-evolutionary algorithm with spatial graph-like structure and predator-prey model for multi-objective optimization [13]. Deb introduced modified algorithm in which predators eliminated preys not only on the basis 
of one criteria but on the basis of the weighted sum of all criteria [5]. Li proposed other modifications to this algorithm [14]. The main difference was that not only predators were allowed to migrate within the graph but also preys could do it. The model of co-operative co-evolution was also applied to multi-objective optimization ([12]).

Sexual selection results from co-evolution of female mate choice and male displayed trait where females evolve to reduce direct costs associated with mating and keep them on optimal level and males evolve to attract females to mating (sexual conflict) [9]. Sexual selection is considered to be one of the ecological mechanisms responsible for biodiversity and sympatric speciation [9].

All the works on sexual selection mechanism for multi-objective evolutionary algorithms were focused on using this mechanism for maintaining population diversity. Allenson proposed genetic algorithm with sexual selection for multi-objective optimization in which the number of sexes was the same as the number of criteria of the given problem [1]. Lis and Eiben proposed multi-sexual genetic algorithm (MSGA) for multiobjective optimization [15] in which also one sex for each criterion was used. They used special multi-parent crossover operator and child had the same sex as the parent that provided most of genes. Bonissone and Subbu continued work on Lis and Eiben's algorithm. They proposed additional mechanisms for determining the sex of offspring (random and phenotype-based) [3].

Co-evolution of species and sexes is the biological mechanism responsible for biodiversity and sympatric speciation. However the application of such mechanisms in evolutionary multi-objective algorithms is still the subject of ongoing research and an open issue.

\section{Introducing Flock-Based Operators into Evolutionary Multi-agent System}

Assuming the classical structure of evolutionary multi-agent system, one of the way for maintaining population diversity and, in the context of multi-objective optimization, for improving the quality of the Pareto frontier approximation consists in introducing to the system so-called flock operators i.e. (in the simplest case) creating new flock/dividing flock into two (n) flocks and merging two (n) flocks into one flock. Taking into account multi-objective optimization goals—-such operators can be realized as follows. During meetings with agents located in the same flock agent gathers (partial) knowledge about its distance (in the decision variable space or in the objective space) from another agents. Then, if such (partial) average distance to another agents is greater than configured parameter(s) (to be precise, in realized system this parameter is changing adaptively) — agent can make a decision about creating new flock (i.e. dividing particular flock into two flocks). After making such a decision agent creates a new flock, migrates to this new flock from the "old" one and then initializes its new flock. "Initialization" process consists in cloning itself and mutating (with small range e.g. by mutating the least significant genes) cloned descendants. The small range of mutation ensures - or increases the probability of — sampling the agent's neighborhood—what 
is very desirable since agent creating new flock stands out in its "original" flock as "strange" agent-i.e. agent representing poorly sampled (at least by this very flock) area of search space. Decision about the number of new cloned descendants created by agent is an autonomous agent's decision of course but it should ensure that flock does not become extinct too early and on the other hand that there do not exist in the system too many similar agent 1 . In the simplest case eliminating/merging flocks operator can be realized as follows: two flocks are being merged if their centers of gravity are located closer than configured value and the difference between their radiuses is smaller than given parameter (both of these parameters can change adaptively).

a)
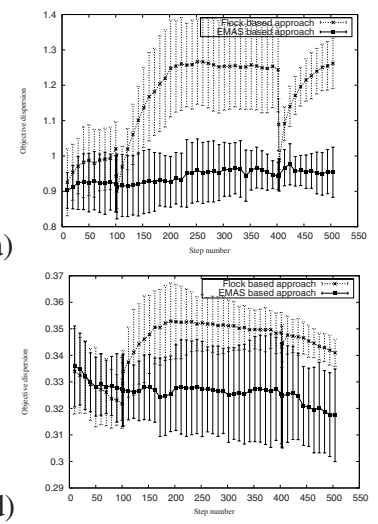

b)

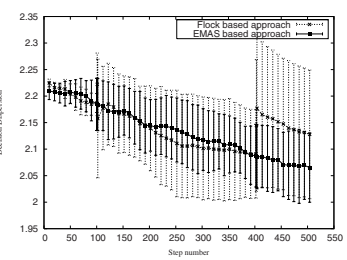

e)

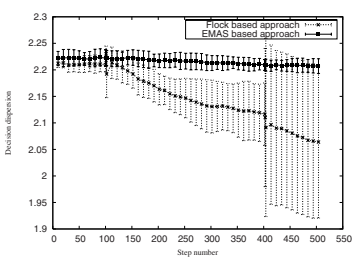

c)

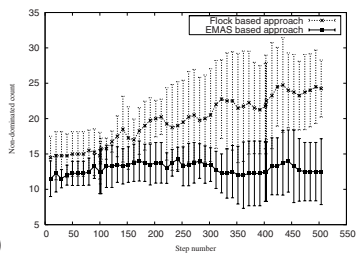

f)

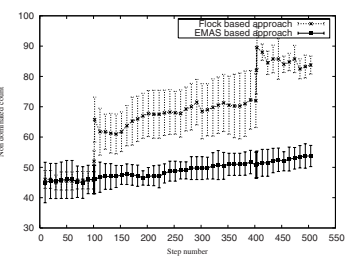

Fig. 1. Selected characteristics: objective dispersion (a,d), decision dispersion (b,e), number of non-dominated individuals (c,f) obtained during solving ZDT-1 (a,b,c) and ZDT-2 (d,e,f) problems

To present the influence of proposed mechanism(s)—during experiments presented in fig. 1 and in fig. 2 2 operator of creating new flocks was performed in 100th and 400th step. Presented in mentioned figures measures should be interpreted as follows: objective dispersion represents the average-measured in the objective space-distance among individuals, decision dispersion represents the average-measured in the space of decision variable - distance among individuals. As one may notice, introducing flock operators influences very positively on maintaining population diversity (see fig. 1a, b, d, e and fig. 2a, b, d, e) and in the consequence on the quality of obtained Pareto frontier approximation (see fig.1k, f and fig.2k, f). Because of the space limitation there are presented in fig. 1 and in fig. 20nly characteristics related to the number of non-dominated solutions found by flock-based and "classical" EMAS for confirming (to some extent) that — at least during solving Zitzler problems ([19]) — flock based approach allows for obtaining much more numerous Pareto set in the comparison to the classical EMAS approach.

\footnotetext{
${ }^{1}$ In tests presented below this value varies from twelve to eighteen.

${ }^{2}$ In presented results below the center of gravity is measured as the arithmetic mean of objective function values of all flock members.
} 
a)
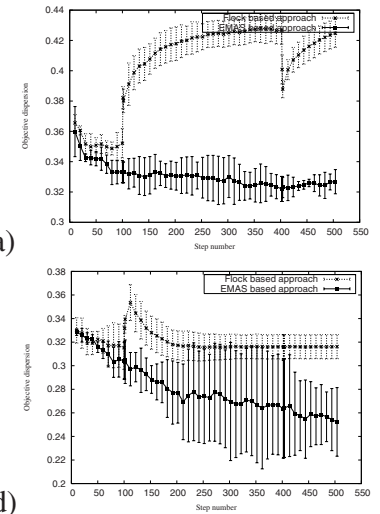

b)

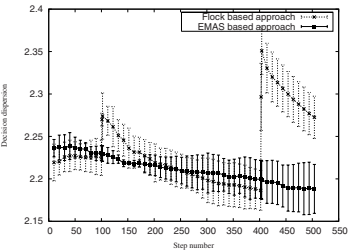

e)

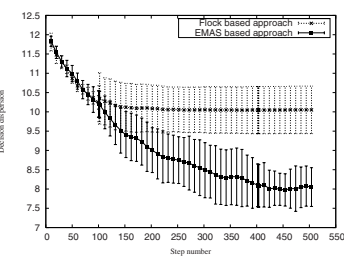

c)

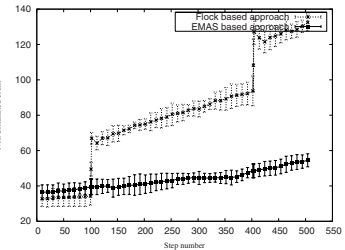

f)

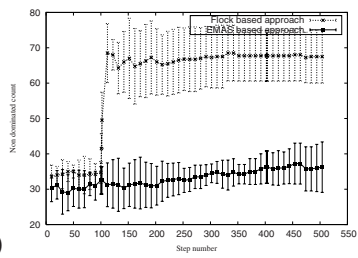

Fig. 2. Selected characteristics: objective dispersion (a, d), decision dispersion (b, e), number of non-dominated individuals (c, f) obtained during solving ZDT-3 (a, b, c) and ZDT-4 (d, e, f) problems

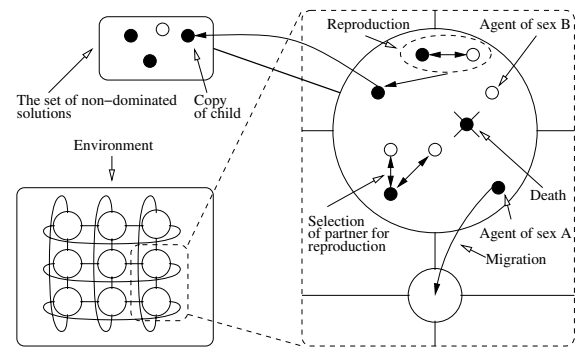

Fig. 3. CoEMAS with co-evolving sexes

\section{Sexual Selection as a Technique for Maintaining Population Diversity in CoEMAS for Multi-objective Optimization}

In order to maintain population diversity in systems based on model of co-evolution in multi-agent system (CoEMAS), mechanisms based on co-evolutionary interactions of species and sexes may be used. Such mechanisms include, for example, host-parasite, predator-prey, or co-operative co-evolution of species. Another way to maintain useful diversity is to apply sexual selection mechanism-resulting system is the CoEMAS with sexual selection (SCoEMAS, see fig. 3).

The mechanisms used in this system include: co-evolution of sexes, and sexual selection based on Pareto domination. All agents live within the environment, which has the graph-like structure. The number of sexes corresponds with the number of criteria (each sex has the criteria assigned to it and agents that belong to that sex are evaluated with the assigned criteria). There is one resource defined in the system. The resource can be possessed by the agents and the environment (there is closed circulation of resource 
in the system). This resource is distributed (proportionally to the fitness values of the agents) by each node of the graph among the agents that are located in that node.

a)

\begin{tabular}{|c|c|}
\hline Population size & 500 \\
\hline Chromosome length & 10 \\
\hline External set size & 256 \\
\hline Crossover probability & 0,3 \\
\hline Mutation probability & 0,2 \\
\hline
\end{tabular}

\begin{tabular}{|c|c|c|c|c|c|c|c|}
\hline \multirow{2}{*}{$\sigma$} & \multirow{2}{*}{ Metrics } & \multicolumn{2}{|c|}{ SPEA } & \multicolumn{2}{c|}{ NSG } & \multicolumn{2}{c|}{ CoEMAS } \\
\cline { 3 - 9 } & & $\begin{array}{l}\text { Obayashi } \\
\text { problem }\end{array}$ & $\begin{array}{c}\text { Tamaki } \\
\text { problem }\end{array}$ & $\begin{array}{c}\text { Obayashi } \\
\text { problem }\end{array}$ & $\begin{array}{c}\text { Tamaki } \\
\text { problem }\end{array}$ & $\begin{array}{c}\text { Obayashi } \\
\text { problem }\end{array}$ & $\begin{array}{c}\text { Tamaki } \\
\text { problem }\end{array}$ \\
\hline \multirow{3}{*}{ c) } & $M_{1}$ & 0.08 & 0.001 & 0.003 & 0.10 & 0.011 & 0.15 \\
\cline { 2 - 8 } & $M_{3}$ & 1.69 & 1.50 & 1.81 & 0.64 & 2.01 & 0.83 \\
\hline 0,05 & $M_{2}$ & 1.75 & 1.49 & 1.84 & 0.41 & 1.37 & 0.39 \\
\hline 0,2 & $M_{2}$ & 3.47 & 5.80 & 1.58 & 5.20 & 1.64 & 5.98 \\
\hline 0,6 & $M_{2}$ & 2.38 & 18.67 & 4.38 & 1.09 & 4.03 & 2.24 \\
\hline
\end{tabular}

\begin{tabular}{|c|c|c|c|c|c|c|c|}
\hline \multicolumn{6}{|c|}{ Population size } & \multicolumn{2}{|c|}{100} \\
\hline \multicolumn{6}{|c|}{ Chromosome length } & \multicolumn{2}{|l|}{8} \\
\hline \multicolumn{6}{|c|}{ External set size } & \multicolumn{2}{|c|}{64} \\
\hline \multicolumn{6}{|c|}{ Crossover probability } & \multicolumn{2}{|c|}{0,1} \\
\hline \multicolumn{6}{|c|}{ Mutation probability } & \multicolumn{2}{|c|}{0,1} \\
\hline \multirow{4}{*}{$\sigma$} & \multirow[b]{2}{*}{ Metrics } & \multicolumn{2}{|c|}{ SPEA } & \multicolumn{2}{|c|}{ NSGA } & \multicolumn{2}{|c|}{ CoEMAS } \\
\hline & & \begin{tabular}{c|}
$\begin{array}{c}\text { Obayashi } \\
\text { problem }\end{array}$ \\
\end{tabular} & $\begin{array}{l}\text { Tamaki } \\
\text { problem }\end{array}$ & \begin{tabular}{|c}
$\begin{array}{c}\text { Obayashi } \\
\text { problem }\end{array}$ \\
\end{tabular} & $\begin{array}{l}\text { Tamaki } \\
\text { problem }\end{array}$ & $\begin{array}{c}\text { Obayashi } \\
\text { problem }\end{array}$ & $\begin{array}{l}\text { Tamaki } \\
\text { problem }\end{array}$ \\
\hline & $M_{\mathrm{t}}$ & 0.13 & 0.32 & \begin{tabular}{|l|}
0.44 \\
\end{tabular} & 0.0 & 0.56 & 0.32 \\
\hline & $M_{3}$ & 0.74 & 1.13 & 1.96 & 0.39 & 2.00 & 0.62 \\
\hline 0,05 & $M_{2}$ & 0.55 & 1.28 & 2.55 & 0.15 & 2.79 & 0.29 \\
\hline 0,2 & $M_{2}$ & 4.38 & 7.17 & 3.95 & 2.98 & 3.88 & 3.14 \\
\hline 0,6 & $M_{2}$ & 0.54 & 14.29 & 7.63 & 2.11 & 8.21 & 2.87 \\
\hline
\end{tabular}

Fig. 4. Comparison of the proposed CoEMAS with sexual selection, SPEA and NSGA algorithms according to the $M_{1}, M_{2}$ and $M_{3}$ metrics (table a includes selected configuration parameters for results presented in table $\mathrm{c}$, and table $\mathrm{b}$ includes parameters for results presented in table d)

Each time step, the agents can migrate within the environment (they lose some resource during the migration). The agent can migrate only to the node connected with the one within which it is located. The agent chooses the node to which it will migrate on the basis of the amount of resource of that node. When the agent is ready for reproduction (i.e. the amount of its resource is above the given level) it sends the information to the agents of other sexes located within the same node. The other agents can response to this information when they are also ready for reproduction. Next, the agent which initiated the reproduction process chooses one (or more-it depends on the number of sexes in the system) of the agents of opposite sex on the basis of the amounts of their resources (the probability of choosing the agent is proportional to the amount of its resource). The offspring is created with the use of intermediate recombination and Gaussian mutation [2]. Next, the child is compared to the individuals from the non-dominated individuals set of the node in which parents and child are located. If none of the individuals from this set is dominating the child then the child is copied to the set (all individuals dominated by the child are removed from the set).

First experiments, which results are presented in this section, were aimed at investigating if SCoEMAS can be applied to multi-objective optimization problems and whether it works properly (agents do not die off). Proposed co-evolutionary multi-agent system with sexual selection mechanism for multi-objective optimization has been tested using, inter alia, Tamaki and Obayashi test functions [18]. Additionally, results obtained with the use of SCoEMAS was compared to those obtained by "classical" evolutionary algorithms for multi-objective optimization: niched-pareto genetic algorithm (NPGA) [5] and strength pareto evolutionary algorithm (SPEA) [19].

To compare proposed approach with implemented classical algorithms three metrics $M_{1}, M_{2}$, and $M_{3}$ ([19]) were used. These metrics are defined as follows. If $A \subseteq X$ denotes a non-dominated set, $\sigma \geq 0$ denotes appropriately chosen neighborhood parameter and $\|\cdot\|$ denotes the given distance metric - then three functions $M_{1}(A), M_{2}(A)$ and $M_{3}(A)$ can be introduced to asses the quality of $A$ regarding the decision space: 
$M_{1}(A)=\frac{1}{|A|} \sum_{a \in A} \min \left\{\|a-x\| \mid x \in X_{p}\right\}$ (the average distance to the Pareto optimal set $\left.X_{p}\right), M_{2}(A)=\frac{1}{|A-1|} \sum_{a \in A}|\{b \in A \mid\|a-b\|>\sigma\}|$ (the distribution in combination with the number of non-dominated solutions found), and $M_{3}(A)=\sqrt{\sum_{i=1}^{N} \max \left\{\left\|a_{i}-b_{i}\right\| \mid a, b \in A\right\}}$ (the spread of non-dominated solutions over the set $A, N$ is the number of objectives).

Presented results (fig. (4) show that SPEA is the best of all compared algorithms. It turned out that proposed SCoEMAS with sexual selection mechanism can be used for multi-objective problems however more research is needed to obtain better results. The fact that results were worse than in the case of classical evolutionary multi-objective algorithms results from the tendency to maintain high population diversity what could be very useful in the case of hard dynamic and multi-modal multi-objective problems (as defined by Deb [4]).

\section{Conclusions}

Maintaining population diversity is one of the main problems in some applications of EAs-especially in multi-modal optimization, multi-objective optimization and adaptation in dynamic environments. In the case of multi-objective optimization problems the loss of population diversity may result in locating only some parts of Pareto frontier or locating a local Pareto frontier instead of the global one in the case of multi-modal multi-objective problems.

In this paper overview of selected techniques and algorithms for maintaining population diversity in (co-)evolutionary multi-agent systems for multi-objective optimization were presented. Proposed mechanisms worked very well from maintaining population diversity (and in the consequence improving the quality of the Pareto frontier approximation) point of view. It is worth to mention in this place that presented flock-based operators as well as co-evolutionary approach with sexual selection are only selected examples of the whole range of mechanisms that can be easily introduced into (co-)evolutionary multi-agent system and that can significantly improve the quality of obtained solutions. Other mechanisms and models such as: semi-elitist evolutionary multi-agent system, distributed frontier crowding, co-evolutionary multi-agent system with host-parasite model, co-evolutionary multi-agent system with predator-prey model should be mentioned, but because of the space limitation they are omitted in this paper. Of course, further research is needed in order to improve proposed mechanisms. It seems that full potential abilities of these systems could be fully observed in the case of hard multi-modal multi-objective problems in which many local Pareto frontiers exist. The future research will also include the application of other co-evolutionary mechanisms like, for example, co-operative co-evolution.

\section{References}

1. R. Allenson. Genetic algorithms with gender for multi-function optimisation. Technical Report EPCC-SS92-01, Edinburgh Parallel Computing Centre, Edinburgh, Scotland, 1992.

2. T. Bäck, D. Fogel, and Z. Michalewicz, editors. Handbook of Evolutionary Computation. IOP Publishing and Oxford University Press, 1997. 
3. S. Bonissone and R. Subbu. Exploring the pareto frontier using multi-sexual evolutionary algorithms: An application to a flexible manufacturing problem. Technical Report 2003GRC083, GE Global Research, 2003.

4. K. Deb. Multi-objective genetic algorithms: Problem difficulties and construction of test problems. Evolutionary Computation, 7(3):205-230, 1999.

5. K. Deb. Multi-Objective Optimization using Evolutionary Algorithms. John Wiley \& Sons, 2001.

6. R. Dreżewski. A model of co-evolution in multi-agent system. In V. Mařík, et al., editor, Multi-Agent Systems and Applications III, volume 2691 of LNCS, pages 314-323, Berlin, Heidelberg, 2003. Springer-Verlag.

7. R. Dreżewski and L. Siwik. Multi-objective optimization using co-evolutionary multi-agent system with host-parasite mechanism. In V. N. Alexandrov, et al., editor, Computational Science - ICCS 2006, volume 3993 of Lecture Notes in Computer Science, pages 871-878, Berlin, Heidelberg, 2006. Springer-Verlag.

8. C. Fonseca and P. Fleming. Genetic algorithms for multiobjective optimization: Formulation, discussion and generalization. In Genetic Algorithms: Proceedings of the Fifth International Conference, pages 416-423. Morgan Kaufmann, 1993.

9. S. Gavrilets. Models of speciation: what have we learned in 40 years? Evolution, 57(10):2197-2215, 2003.

10. P. Hajela and C. Lin. Genetic search strategies in multicriterion optimal design. In Structural optimization 4, pages 99-107, 1992.

11. J. Horn, N. Nafpliotis, and D. E. Goldberg. A niched pareto genetic algorithm for multiobjective optimization. In Proceedings of the First IEEE Conference on Evolutionary Computation, pages 82-87, Piscataway, New Jersey, 1994. IEEE Service Center.

12. A. Iorio and X. Li. A cooperative coevolutionary multiobjective algorithm using nondominated sorting. In K. Deb, et al., editor, Genetic and Evolutionary Computation - GECCO 2004, volume 3102-3103 of LNCS, pages 537-548. Springer-Verlag, 2004.

13. M. Laumanns, G. Rudolph, and H.-P. Schwefel. A spatial predator-prey approach to multiobjective optimization: A preliminary study. In A. Eiben, et al., editor, Parallel Problem Solving from Nature - PPSN V, volume 1498 of LNCS. Springer-Verlag, 1998.

14. X. Li. A real-coded predator-prey genetic algorithm for multiobjective optimization. In C. M. Fonseca, et al., editor, Evolutionary Multi-Criterion Optimization, Second International Conference (EMO 2003), Proceedings, volume 2632 of LNCS. Springer-Verlag, 2003.

15. J. Lis and A. E. Eiben. A multi-sexual genetic algorithm for multiobjective optimization. In T. Fukuda and T. Furuhashi, editors, Proceedings of the Third IEEE Conference on Evolutionary Computation, pages 59-64, Piscataway NJ, 1996. IEEE Press.

16. S. W. Mahfoud. Niching methods for genetic algorithms. PhD thesis, University of Illinois at Urbana-Champaign, Urbana, IL, USA, 1995.

17. N. Srinivas and K. Deb. Multiobjective optimization using nondominated sorting in genetic algorithms. Evolutionary Computation, 2(3):221-248, 1994.

18. D. A. Van Veldhuizen. Multiobjective Evolutionary Algorithms: Classifications, Analyses, and New Innovations. PhD thesis, Graduate School of Engineering of the Air Force Institute of Technology Air University, 1999.

19. E. Zitzler. Evolutionary algorithms for multiobjective optimization: methods and applications. PhD thesis, Swiss Federal Institute of Technology, Zurich, 1999. 